\title{
NONLINEAR PROPERTIES OF WAVEGUIDES WITH TWISTED NEMATIC LIQUID CRYSTAL
}

\author{
M.A. KARPIERZ \\ Faculty of Physics, Warsaw University of Technology \\ Koszykowa 75, 00-662 Warszawa, Poland
}

\begin{abstract}
Optical reorientational nonlinearity in twisted nematic liquid crystalline planar waveguide is analyzed theoretically. It is shown that optical nonlinearity for guided modes in analyzed waveguides can be approximated by saturation nonlinearity. Predicted nonlinearity is large enough to observe effects like spatial solitons formation with milliwatts of light power.
\end{abstract}

PACS numbers: 42.65.Wi, 42.70.Df

\section{Introduction}

Liquid crystals possess many unique physical, optical, and electro-optical properties which cause that they are important materials in numerous technical applications and they are very interesting medium for many investigations in basic research. In the nematic phase, the correlation among liquid crystalline molecules is very strong because of the high anisotropy and the collective behavior of the molecules. This is responsible for the fact that liquid crystal molecules can easily reorient even with a very low applied field. The molecular reorientation due to interaction with the electric field of the light wave modifies the local birefringence axis of liquid crystal and it is the basis of the optical reorientational nonlinearity $[1,2]$. The reorientational nonlinearity in liquid crystalline waveguides leads to numerous effects not observed in another types of nonlinearity. Among others there are obtained the threshold and optical bistable effects resulting in nonlinear refractive index changes, strong dependence on light polarization as well as possibility of controlling the nonlinearity by external electric or magnetic fields. Theoretically analyzed unique properties of liquid crystalline nonlinear wa veguides have been also confirmed in recently reported experiments with liquid crystalline waveguides [3-8]. 


\section{Waveguide modes}

In this paper the detailed analysis of the nonlinear properties in a planar waveguide filled with twisted nematic liquid crystal (see Fig. 1) is presented. Nematic liquid crystal is an anisotropic medium with axis of birefringence parallel to the mean direction of the molecules orientation, defined by the director $n$. In analyzed nematic orientation the liquid crystalline molecules are assumed to be placed in $y z$ plane and they are twisted around $z$ axis. The orientation of molecules can be described by angle $\theta$ measured according to the $y$ axis: $n=(0, \cos \theta, \sin \theta)$. Then the electric permittivity tensor in twisted nematics has the form

$$
\epsilon=\left(\begin{array}{ccc}
\epsilon_{\perp} & 0 & 0 \\
0 & \epsilon_{\perp}+\Delta \epsilon \cos ^{2} \theta & \Delta \epsilon \cos \theta \sin \theta \\
0 & \Delta \epsilon \cos \theta \sin \theta & \epsilon_{\perp}+\Delta \epsilon \sin ^{2} \theta
\end{array}\right),
$$

where $\Delta \epsilon=\epsilon_{\|}-\epsilon_{\perp}$ is an optical anisotropy, $\epsilon_{\perp}=n_{\mathrm{o}}^{2}$ is an ordinary and $\epsilon_{\|}=n_{\mathrm{e}}^{2}$ is an extraordinary electric permittivity. For guided electromagnetic wave in planar waveguide it is assumed that the electric and magnetic fields do not depend on $y$ coordinate and they depend on $z$ coordinate as a function $\exp (-\mathrm{i} k N z)$, where $k$ is a wavevector length and $N$ is an effective refractive index of the guided mode. Then, the Maxwell equations have the form

$$
\begin{aligned}
& \frac{\partial}{\partial x} E_{y}=-\mathrm{i} k Z H_{z}, \\
& -\mathrm{i} Z \frac{\partial}{\partial x} H_{z}=k\left(N^{2}-\epsilon_{y y}\right) E_{y}-k \epsilon_{y z} E_{z}, \\
& Z H_{x}=-N E_{y}, \\
& \frac{\partial}{\partial x} E_{z}=-\mathrm{i} k\left(\frac{N^{2}}{\epsilon_{\perp}}-1\right) Z H_{y}, \\
& -\mathrm{i} Z \frac{\partial}{\partial x} H_{y}=k \epsilon_{y z} E_{y}+k \epsilon_{z z} E_{z}, \\
& E_{x}=\frac{N}{\epsilon_{\perp}} Z H_{y},
\end{aligned}
$$

where $Z=c \mu_{0}$ is the free space impedance and $\epsilon_{a b}$ are components of the electric permittivity tensor (1).

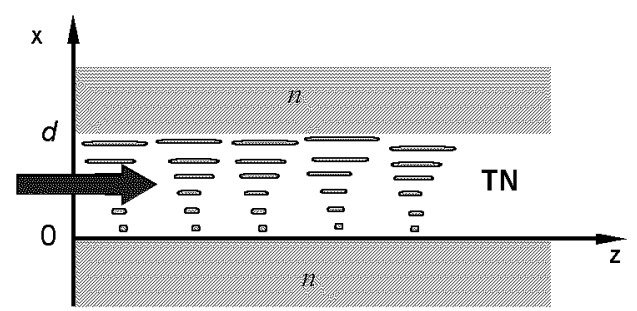

Fig. 1. Schematic drawing of analyzed waveguide with twisted nematics (TN). 
In isotropic substrate and cover surrounding the liquid crystalline film, the electrical permittivity becomes $\epsilon=n^{2}$, where $n$ is a refractive index of the medium. Then the electromagnetic wave in isotropic cover and substrate splits into two separate solutions corresponding to TE-like and TM-like fields, respectively. TE fields are governed by Eqs. (2)-(4) with $\epsilon_{y z}=0$ and $\epsilon_{y y}=n^{2}$ and for the guided modes (with $N>n$ ) they are equal to

$$
\begin{aligned}
& E_{y}(x)=A_{ \pm} \exp ( \pm \gamma x) \\
& Z H_{z}(x)= \pm \mathrm{i} A_{ \pm} \frac{\gamma}{k} \exp ( \pm \gamma x)
\end{aligned}
$$

where $\gamma=k \sqrt{N^{2}-n^{2}}$. Consequently the TM-like fields are described by Eqs. (5)-(7) with $\epsilon_{y z}=0$ and $\epsilon_{z z}=\epsilon_{\perp}=n^{2}$ and they have the solution

$$
\begin{aligned}
& Z H_{y}(x)=\mathrm{i} B_{ \pm} \exp ( \pm \gamma x), \\
& E_{z}(x)= \pm B_{ \pm} \frac{\gamma}{k n^{2}} \exp ( \pm \gamma x) .
\end{aligned}
$$

The sign " + " in solutions $(8)-(11)$ corresponds to fields in substrate $(x<0)$ vanishing for $x \rightarrow-\infty$ and the sign "-" corresponds to fields in the cover for $(x>d)$ vanishing for $x \rightarrow \infty$. Solution in the form of guided mode in the liquid crystalline film does not exist as an analytical formula for arbitrary twisted geometry. Therefore the field distribution inside the waveguide film should be obtained by direct numerical integration of Eqs. (2)-(7). The solving procedure requires to fulfill boundary conditions at $x=0$ and $x=d$, this means that only one from four constants $A_{ \pm}$and $B_{ \pm}$in Eqs. (8)-(11) is arbitrary.

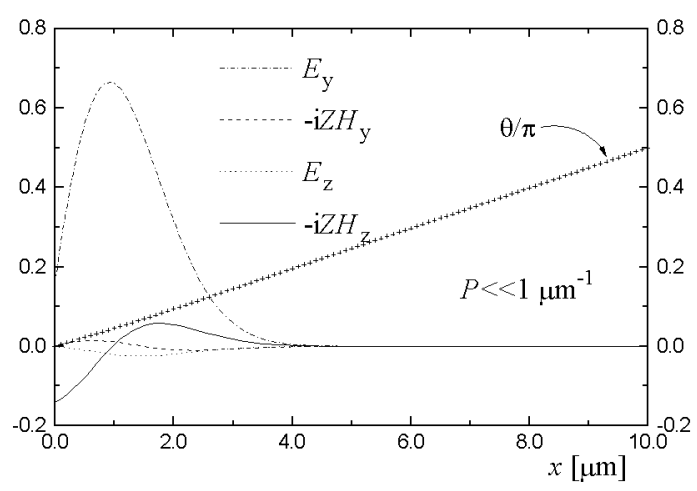

Fig. 2. Electric and magnetic field components (in arbitrary units) distribution in guided mode corresponding to orientation angle $\theta$ showed as a line with crosses.

Figure 2 presents the electric and magnetic field components obtained for fundamental guided mode in liquid crystalline film with linearly twisted nematics: $\theta(x)=\pi x / 2 d$. In this paper the following parameters were taken for numerical calculations: light wavelength $\lambda=842 \mathrm{~nm}$, thickness of the liquid crystalline film $d=10 \mu \mathrm{m}$, refractive index of waveguide cover and substrate corresponding to silica glass $n=1.45$, refractive indices of liquid crystal corresponding to 4 -trans-4'- $n$-hexyl-cyclohexyl-isothiocyanatobenzene (6CHBT): ordinary $n_{\circ}=1.52$ and extraordinary $n_{\mathrm{e}}=1.69$. The field of guided mode in Fig. 2 
is confined close to the boundary $x=0$. This is caused by the fact that the fundamental guided mode (with the largest effective refractive index $N$ ) has a dominating electric field $E_{y}$ and for this polarization the largest value of refractive index is close to the border $x=0$ (where liquid crystal molecules are parallel to the $y$ axis). Note that according to Eqs. (2)-(7) magnetic fields $H_{y}$ and $H_{z}$ as well as component $E_{x}$ (proportional to $H_{y}$ ) are shifted by $\pi / 2$ in phase to the phase of components $E_{y}, E_{z}$, and $H_{x}$. Therefore in further analysis it will be assumed that field distribution components $E_{y}, E_{z}$, and $H_{x}$ are real and components $H_{y}, H_{z}$, and $E_{x}$ are imaginary.

\section{Reorientational nonlinearity}

At a given temperature nematic liquid crystal molecules fluctuate around the mean direction defined by the director $\boldsymbol{n}$. The distortion of the molecular alignment corresponds to the free energy density [1]

$$
f_{\mathrm{F}}=\frac{K_{11}}{2}(\nabla n)^{2}+\frac{K_{22}}{2}(n \cdot \nabla \times \boldsymbol{n})^{2}+\frac{K_{33}}{2}(\boldsymbol{n} \times \nabla \times \boldsymbol{n})^{2},
$$

where $K_{i i}$ are elastic constants for three different deformations: splay $(i=1)$, twist $(i=2)$, and bend $(i=3)$. External electric or magnetic fields create a torque on the molecules and they change the mean orientation of the liquid crystal. For optical frequencies the interaction with magnetic field can be neglected and light waves interact with nematic liquid crystals through the electric field. The orientation-dependent term in the interaction energy density between the electric field and liquid crystal molecules is equal to

$$
f_{\mathrm{opt}}=-\frac{\epsilon_{0} \Delta \epsilon}{2}\left\langle(\boldsymbol{n} \cdot \boldsymbol{E})^{2}\right\rangle
$$

Liquid crystalline molecules tend to be parallel to the electric field. Because the reorientation is slow in comparison to the electromagnetic wave period, the molecules tend to lie parallel to the mean direction of electric field. Especially in analyzed configuration, when $E_{x}$ component is shifted in phase by $\pi / 2$ to the $E_{y}$ and $E_{z}$ and is much lower than $E_{y}\left(E_{y} \gg E_{x}\right)$ the molecules are reoriented in $y z$ plane. This means that due to the reorientation the twisted geometry is still preserved, i.e. the orientation is described by the angle $\theta(x)$ and the electrical permittivity tensor has the form (1). As a consequence only the twist deformation takes place and the total free energy density composed of the deformation energy and the interaction energy $f=f_{\mathrm{F}}+f_{\text {opt }}$ is equal to

$$
f=\frac{1}{2} K_{22}\left(\frac{\mathrm{d} \theta}{\mathrm{d} x}\right)^{2}-\frac{\epsilon_{0} \Delta \epsilon}{4}\left(E_{y}^{2} \cos ^{2} \theta+E_{z}^{2} \sin ^{2} \theta+2 E_{y} E_{z} \cos \theta \sin \theta\right) \text {. }
$$

The total free energy $\int_{0}^{d} f \mathrm{~d} x$ fulfills a minimization procedure which leads to the Euler-Lagrange equation in the form

$$
\frac{\partial}{\partial x} \frac{\partial f}{\partial\left(\frac{\mathrm{d} \theta}{\mathrm{d} x}\right)}-\frac{\partial f}{\partial \theta}=0
$$


which finally gives the equation

$$
\frac{\mathrm{d}^{2} \theta}{\mathrm{d} x^{2}}+\frac{\epsilon_{0} \Delta \epsilon}{4 K_{22}}\left[\left(E_{z}^{2}-E_{y}^{2}\right) \sin 2 \theta+2 E_{z} E_{y} \cos 2 \theta\right]=0 .
$$

Solution of Eq. (16) should fulfill the boundary condition at $x=0$ and $x=d$. For strong anchoring $\theta(0)$ and $\theta(d)$ are independent of light intensity. In the absence of electric fields and for boundary conditions $\theta(0)=0$ and $\theta(d)=\pi / 2$ the solution is the same as assumed in previous section i.e. $\theta(x)=\pi x / 2 d$. In that case the field distribution presented in Fig. 2 corresponds to the mode with light power too low to introduce reorientation of liquid crystal. For higher power of light guided in the waveguide the liquid crystal molecules change their orientation due to the interaction with electric field and this causes changes of electric permittivity tensor (1). As a result of this reorientational nonlinearity mechanism the fields distribution as well as the effective refractive index $N$ of guided mode are changing. Figure 3 shows the electric and magnetic fields distribution in nonlinear mode for light power large enough to reorient significantly the liquid crystalline molecules. As a light power the normalized parameter is used

$$
P=\frac{\epsilon_{0} \Delta \epsilon Z}{4 K_{22}} \int S_{z} \mathrm{~d} x
$$

where $S_{z}$ is a component of the Poynting vector

$$
S_{z}=\frac{N}{Z}\left(E_{y}^{2}+\frac{1}{\epsilon_{\perp}}\left|Z H_{y}\right|^{2}\right) .
$$

Note that for typical values of elastic constant $K \approx 10^{-11} \mathrm{~N}$ the value of parameter $P=1 \mu \mathrm{m}^{-1}$ corresponds to power density $20 \mathrm{~mW} / \mu \mathrm{m}$.

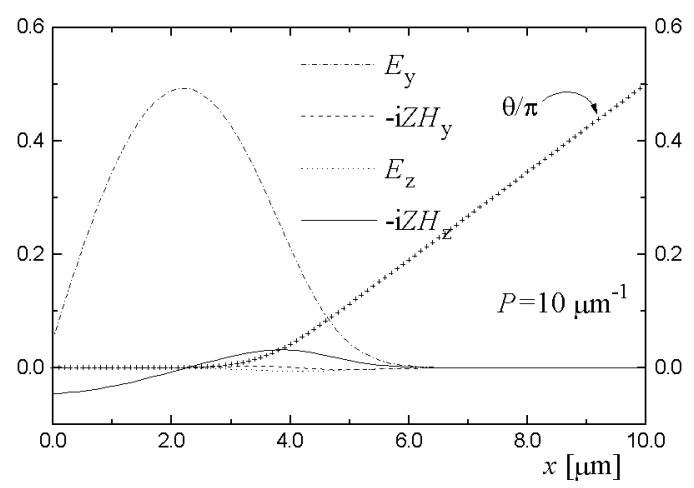

Fig. 3. Electric and magnetic fields distribution in guided mode for power $P=$ $10 \mu \mathrm{m}^{-1}$. Line with crosses shows the distribution of orientation angle $\theta$.

Comparison of Figs. 2 and 3 shows that due to the nonlinear reorientation the center of guided mode is shifting into the center of the guided film. This is more clearly visible in Figs. 4-6, where both electric and magnetic fields and orientation angle distribution across the liquid crystalline film are plotted for increasing values of guided light power. 


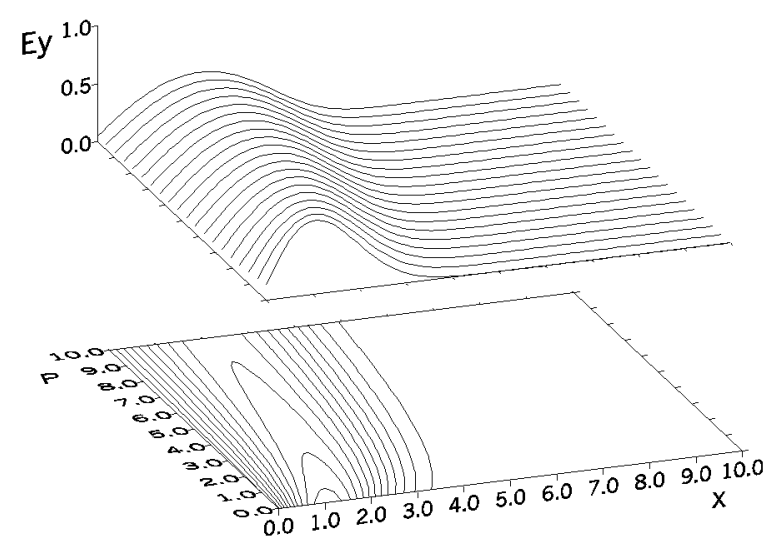

Fig. 4. Changes of electric field $E_{y}$ profile with increasing light power $P$ (in $\mu \mathrm{m}^{-1}$ ) of guided mode ( $x$ in $\mu \mathrm{m}$ ).

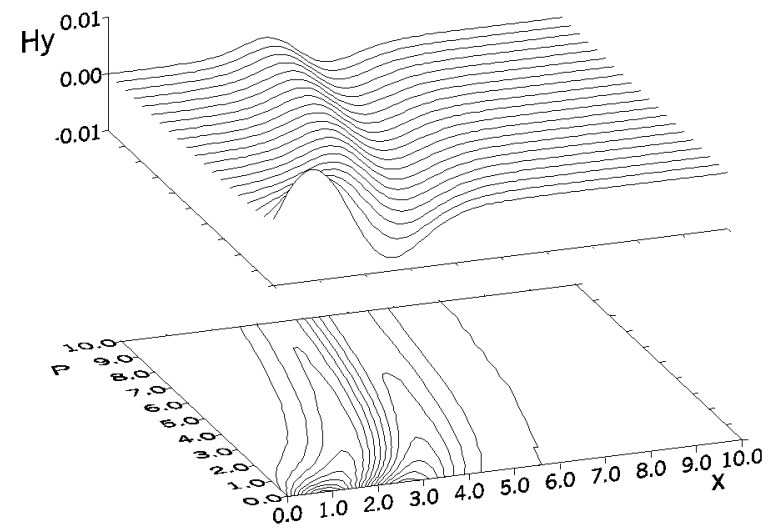

Fig. 5. Changes of magnetic field $H_{y}$ profile with increasing light power $P$ in guided mode.

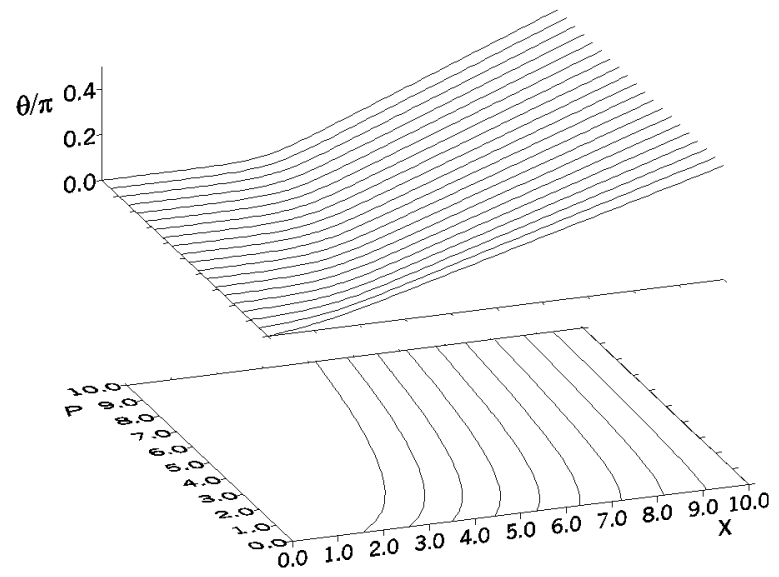

Fig. 6. Orientation angle $\theta$ changes with increasing light power $P$ of nonlinear guided mode. 
The power $P$ used in Figs. 4-6 corresponds to the component perpendicular to front phase of the guided mode. In fact, in analyzed anisotropic waveguide the energy flow direction is different. It is presented in Fig. 7 where the ratio $P_{y} / P$ between the power flow in $y$ direction

$$
P_{y}=-\frac{\epsilon_{0} \Delta \epsilon N}{4 K_{22}} \int\left(E_{y} E_{z}+\frac{Z^{2}}{\epsilon_{\perp}}\left|H_{y} H_{z}\right|\right) \mathrm{d} x
$$

and the power flow in $z$ direction is plotted versus $P$. With increasing light power the orientation of liquid crystal molecules (orientation of birefringence axis) becomes more homogeneous (see Fig. 6) and this causes that the walk-off of energy flow is decreasing. This effect can be observed if instead of planar waveguide mode (infinite light wave) the light beam in a planar waveguide is used. Then with the increase in input light power the bend in propagation of light beam should change simultaneously with self-focusing phenomena.

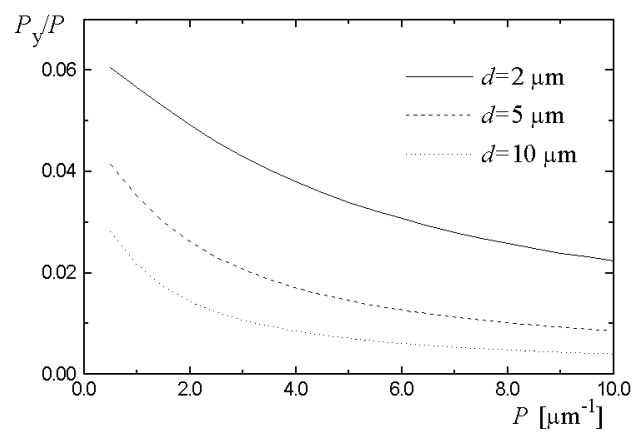

Fig. 7. Fraction of power flow in $y$ direction versus the power guided in $z$ direction for nonlinear modes.

The walk-off of the energy flow does not exist in symmetrical twisted nematic configuration, with boundary conditions $\theta(0)=-\pi / 4, \theta(d)=\pi / 4$. In this configuration for linear case (without reorientation induced by electric field) the orientation distribution has the form $\theta(x)=\pi(x-d / 2) / 2 d$. The field and orientation angle distribution changes with increasing guided power are presented in Figs. 8-10. In this case the changes of the electric and magnetic fields are symmetrical but lower than for previously presented results in Figs. 5 and 6 .

The nonlinear reorientation changes the modal field profile and also the effective refractive index $N$ of the guided mode. The dependence of effective refractive index on the light power is presented in Figs. 11 and 12. Because the analyzed field has dominant $E_{y}$ component, the effective refractive index is mainly determined by $\epsilon_{y y}$ and the liquid crystals reorient to be parallel to $y$ axis. Therefore the initial value of the effective index $N$ for symmetrical configurations is larger than in asymmetrical configurations. In both cases the increase in effective index is saturation-like, where the maximal value of $N$ reached for infinite light power $P$ is determined by effective refractive index in planar orientation of liquid crystal, i.e. for $\theta(x)=0$. In symmetrical configuration this maximal reorientation is 


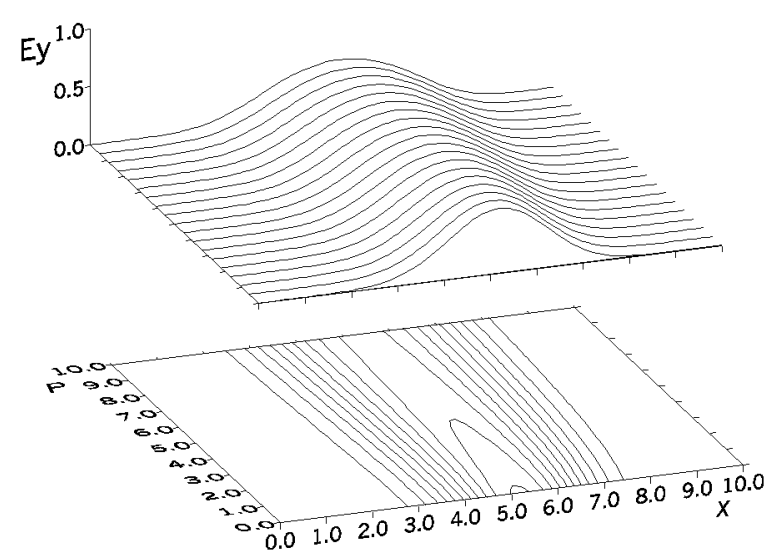

Fig. 8. Electric field $E_{y}$ distribution changes with increasing power $P$ for symmetric twisted nematic configuration $(\theta(0)=\pi / 4, \theta(d)=-\pi / 4)$.

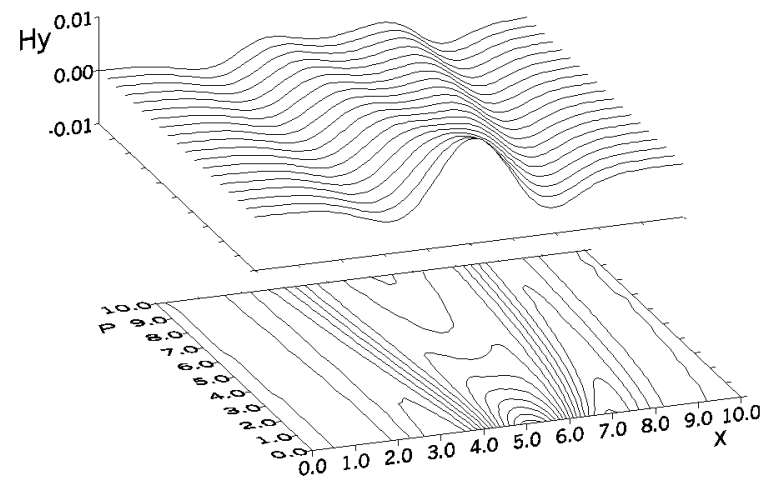

Fig. 9. Changes of magnetic field $H_{y}$ profile with changing the power $P$ for symmetric twisted nematics.

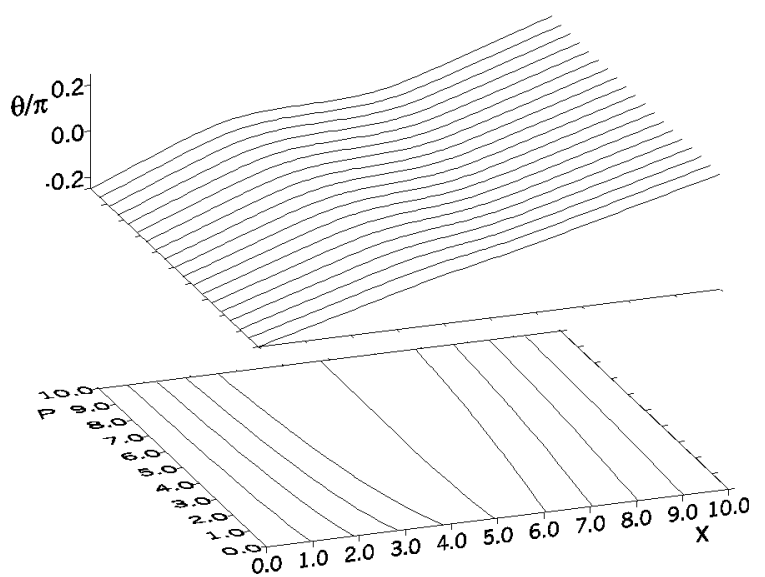

Fig. 10. Changes of the orientation angle $\theta$ with increasing guided power $P$ for symmetric twisted nematics. 


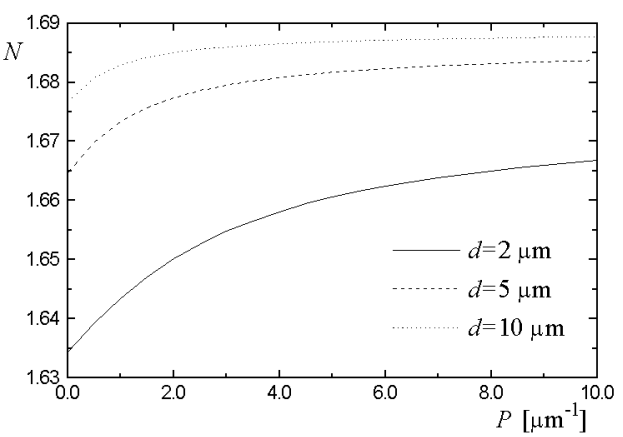

Fig. 11. Effective refractive index $N$ changes versus power $P$ guided in nonlinear mode for different thickness $d$ of the layer with asymmetrical alignment of twisted nematics.

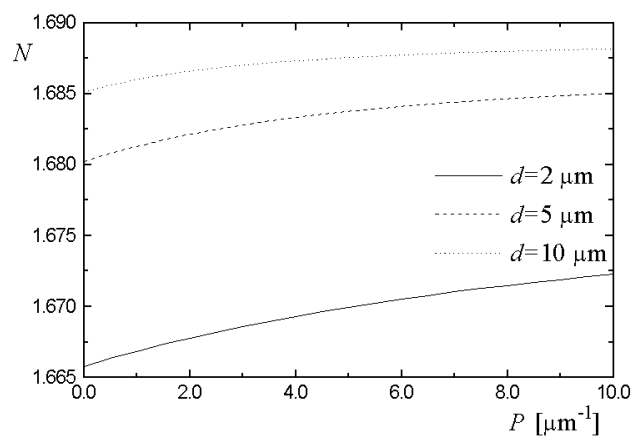

Fig. 12. Effective refractive index $N$ changes versus power $P$ guided in nonlinear mode for symmetrical twisted nematics.

lower than $\Delta \theta= \pm \pi / 4$ and it can take place close to the boundaries i.e. in region where the electrical field is low. In asymmetrical case the maximal reorientation $\Delta \theta \leq \pi / 2$ and it is also large in the central part of the liquid crystalline film. Consequently, the changes of $N$ due to nonlinearity are lower in symmetrical than in asymmetrical configurations. The differences in nonlinear changes for different waveguide thickness are caused by different confinement of electromagnetic field in the guided film.

\section{Simplified model of nonlinearity}

In many applications of nonlinear waveguide optics more important than changes of guided mode profile are changes of the effective refractive index. The calculation of effective index changes due to reorientational nonlinearity presented in previous section requires to solve the set of equations with proper boundary conditions. However, the obtained nonlinearity can be approximated with very high accuracy by an analytical formula. To obtain this formula first it is assumed 
that the liquid crystalline film is reoriented by homogeneous electric field with $E_{z}=0$, and $E_{y}=4 K_{22} \varepsilon / \epsilon_{0} \Delta \epsilon$. With these assumptions Eq. (16) reduces to

$$
\frac{\mathrm{d}^{2} \theta}{\mathrm{d} x^{2}}+\varepsilon^{2} \sin 2 \theta=0
$$

and it has a solution

$$
\sin \left(\theta+\frac{\pi}{2}\right)=\operatorname{sn}\left[\alpha\left(x+x_{0}\right) \mid m\right],
$$

where $\operatorname{sn}(u \mid m)$ is the Jacobi elliptic integral [9] with the modulus $m=2 \varepsilon^{2} / \alpha^{2}$. For the boundary condition $\theta(x=0)=0$ it is obtained that $\operatorname{sn}\left(\alpha x_{0} \mid m\right)=1$ and this allows to determine the relation between integration constants: $\alpha x_{0}=\mathrm{K}(m)$, where $\mathrm{K}(\mathrm{m})$ is the complete elliptic integral of the second kind. Then the solution (21) has the form

$$
\cos \theta=c d(\alpha x \mid m),
$$

where $\operatorname{cd}(\alpha x \mid m)$ is the Jacobi elliptic function.

The reorientation of liquid crystalline molecules changes locally the electric permittivity, which for $E_{y}$ electric field is proportional to $\Delta \epsilon \cos ^{2} \theta$. For guided modes more important than local values are effective changes obtained as an integral over cross-section. Therefore it is convenient to determine the integral $\int_{0}^{d} \cos ^{2} \theta \mathrm{d} x$, from Eq. (22) as

$$
\int_{0}^{d} \cos ^{2} \theta \mathrm{d} x=\frac{1}{\alpha m}[\alpha d-\mathrm{E}(\alpha d \mid m)+m \operatorname{sn}(\alpha d \mid m) \operatorname{cd}(\alpha d \mid m)],
$$

where $\mathrm{E}(u \mid m)$ is an elliptical integral of the second kind. For the orientation at the second boundary $\theta(x=d)=\pi / 2$ it is obtained that $\mathrm{cd}(\alpha d)=0$ and consequently $\alpha d=\mathbf{K}(m)$. Then the modulus $m$ can be calculated from the relation $m \mathrm{~K}^{2}(m)=$ $2 \varepsilon^{2} d^{2}$ and the integral (23) reduces to

$$
\int_{0}^{d} \cos ^{2} \theta \mathrm{d} x=\frac{\mathrm{K}(m)-E[\mathrm{~K}(m) \mid m]}{\mathrm{K}(m) m} d .
$$

Next important approximation is an assumption that reorientation is very small, i.e. because of small value of electric field $\varepsilon \ll$ the modulus of elliptic integrals is small, $m \ll 1$. This allows to reduce elliptic integrals to first elements in expansions into series: $\mathrm{K}(m) \approx \frac{\pi}{2}\left(1+\frac{1}{4} m+\ldots\right)$ and $\mathrm{E}\left(\frac{\pi}{2} \mid m\right) \approx \frac{\pi}{2}\left(1-\frac{1}{4} m+\ldots\right)$. Finally, the simplified form of relation (12) is obtained as follows:

$$
\int_{0}^{d} \cos ^{2} \theta \mathrm{d} x \approx \frac{\mathrm{K}(m)-\mathrm{E}\left(\frac{\pi}{2} \mid m\right)}{\mathrm{K}(m) m} d \approx \frac{2 d}{4+m},
$$

and the effective change of the electrical permittivity between linear case (where $\varepsilon=0$ and $m=0$ ) and nonlinear case is equal to

$$
\delta \epsilon=\frac{1}{d} \int_{0}^{d} \Delta \epsilon\left[\cos ^{2} \theta(\varepsilon=0)-\cos ^{2} \theta(\varepsilon)\right] \mathrm{d} x \approx \frac{\Delta \epsilon}{2} \frac{m}{4+m} .
$$

Because modulus $m$ is roughly proportional to the square of electric field $m \sim \varepsilon^{2}$, 
the relation (26) leads to

$$
\delta \epsilon \sim \frac{\varepsilon^{2}}{1+\varepsilon^{2} / \varepsilon_{0}^{2}},
$$

where $\varepsilon_{0}$ is a constant.

The above relation (27) was obtained for low homogeneous electric field $E_{y}$ in liquid crystals oriented at the boundary $\theta(0)=0$ and $\theta(d)=\pi / 2$. However, it could be expected that for guided mode the behavior is similar and effective refractive index is modified by effective changes due to reorientation $N=N_{0}+\delta \epsilon / 2 N_{0}$. Because instead of square of electric field for guided mode it is more convenient to use light power, finally the approximated form of nonlinearity is proposed in the form

$$
N=N_{0}+N_{2} \frac{P}{1+P / P_{0}}
$$

where power $P_{0}, N_{0}$, and $N_{2}$ are constants determined for given guided mode.

The quality and exactness of approximated formula (28) can be proved in Fig. 13, where the effective index determined numerically is plotted versus power expression $P /\left(1+P / P_{0}\right)$. The value of power saturation $P_{0}$ and nonlinear index $N_{2}$ were fitted numerically independently for different waveguides. The parameters values estimated numerically are presented in Table. In all cases the correlation coefficient for 21 points in linear regression procedure was larger than 0.99969 and standard deviation lower than 0.00024 . Interesting is the fact that the value of $N_{2}$ is almost independent of the film thickness for the analyzed orientation of twisted nematic waveguides.

TABLE

Parameters used for approximation of nonlinearity in saturation form.

\begin{tabular}{c|c|c|c}
\hline \hline & \multicolumn{3}{|c}{$0 \leq \theta \leq \pi / 2$} \\
\hline$d[\mu \mathrm{m}]$ & 2 & 5 & 10 \\
$N_{0}$ & 1.6338 & 1.6645 & 1.6765 \\
$N_{2}[\mu \mathrm{m}]$ & 0.0130 & 0.0151 & 0.0137 \\
$P_{0}\left[\mu \mathrm{m}^{-1}\right]$ & 3.43 & 1.46 & 0.88 \\
$\chi^{2}$ & $6.2 \times 10^{-8}$ & $1.5 \times 10^{-8}$ & $2.8 \times 10^{-9}$ \\
\hline \multicolumn{3}{|c}{$-\pi / 4 \leq \theta \leq \pi / 4$} \\
\hline$d[\mu \mathrm{m}]$ & 2 & 5 & 10 \\
$N_{0}$ & 1.66578 & 1.68019 & 1.68511 \\
$N_{2}[\mu \mathrm{m}]$ & 0.00116 & 0.00131 & 0.00117 \\
$P_{0}[\mu \mathrm{m}-1]$ & 12.8 & 5.92 & 3.55 \\
$\chi^{2}$ & $9.0 \times 10^{-11}$ & $1.9 \times 10^{-10}$ & $1.4 \times 10^{-10}$
\end{tabular}




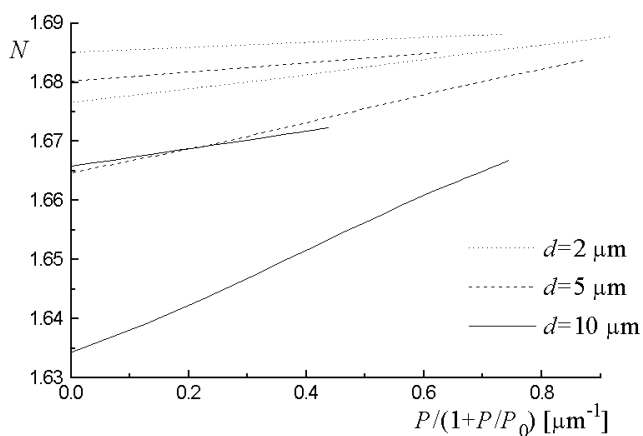

Fig. 13. Effective refractive index versus $P /\left(1+P / P_{0}\right)$ in waveguides with symmetrical configurations (upper lines) and asymmetrical configurations (lower lines) where the values of parameter $P_{0}$ for each line is shown in Table.

\section{Conclusions}

The twisted nematic liquid crystals thin films can be easily applied as a nonlinear planar waveguide. The reorientational nonlinearity for relatively low intensities (for several tens $\mathrm{mW} / \mu \mathrm{m}$ ) induces significant changes in guided mode profile and effective refractive index (up to $\Delta N \approx 0.1$ ). The nonlinearity can be a source of typical nonlinear phenomenon like self-focusing (spatial solitons formation). The unique properties of twisted nematics configuration allows also to change the bend of light beam by changing the light power. This effect can be applied in all-optical switching devices.

The analyzed nonlinearity can be approximated with very high accuracy by saturation nonlinearity. This type of nonlinearity is very well known and observed in other materials $[10,11]$. The estimated value of nonlinear refractive index in analyzed waveguides is larger than in many other materials. The calculated nonlinearity corresponds to that obtained in bulk materials with nonlinear refractive index $n_{2} \approx 10^{-12} \mathrm{~m}^{2} / \mathrm{W}$. The nonlinearity can be additionally enlarged even two orders of magnitude by utilizing the Janossy effect in dye-doped liquid crystals [12]. However, reorientational nonlinearity in liquid crystals is slow and the relaxation time of nonlinear changes is of order of milliseconds.

This work was partially supported by the Committee for Scientific Research under the grant No. 8T11D 01915.

\section{References}

[1] I.C. Khoo, S.T. Wu, Optics and Nonlinear Optics of Liquid Crystals, World Sci., Singapore 1993.

[2] N.V. Tabiryan, A.V. Sukhov, B.Ya. Zeldovich, Mol. Cryst. Liq. Cryst. 136, 1 (1986).

[3] G. Abbate, F. Castaldo, L. De Stefano, Mol. Cryst. Liq. Cryst. 282, 269 (1996). 
[4] M. Warenghem, J.F. Henniot, G. Abbate, Opt. Express 2, 483 (1998).

[5] M. Warenghem, J.F. Henninot, G. Abbate, Mol. Cryst. Liq. Cryst. 320, 207 (1998).

[6] M.A. Karpierz, T.R. Wolinski, M. Swillo, Mol. Cryst. Liq. Cryst. 282, 365 (1996).

[7] M.A. Karpierz, M. Sierakowski, M. Swillo, T.R. Wolinski, Mol. Cryst. Liq. Cryst. 320, 157 (1998).

[8] M. Peccianti, A. De Rossi, G. Assanto, A. De Luca, C. Umeton, I.C. Khoo, Appl. Phys. Lett. 77, 7 (2000).

[9] P.F. Byrd, M.D. Friedman, Handbook of Elliptic Integrals for Engineers and Scientists, Springer-Verlag, New York 1971.

[10] S. Gatz, J. Herrmann, J. Opt. Soc. Am. B 8, 2296 (1991).

[11] H. Hatami-Hanza, P.L. Chu, G.D. Peng, Opt. Quantum Electron. 26, S365 (1994).

[12] I. Janossy, Phys. Rev. E 49, 2957 (1994). 